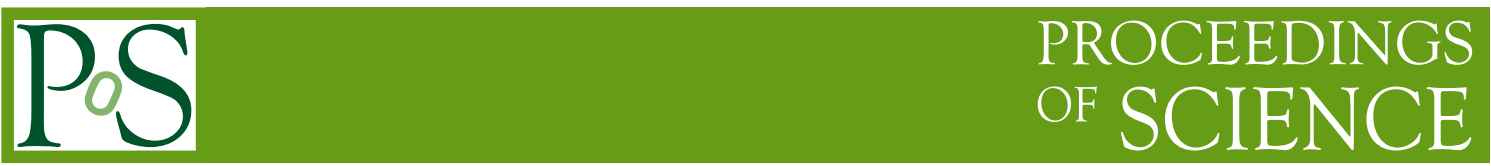

\title{
Tracking Trigger Upgrade Plans for CMS at SLHC
}

\author{
Mark Pesaresi ${ }^{* \dagger}$
}

Imperial College London, UK

E-mail: mark.pesaresi@imperial.ac.uk

The Super-LHC (SLHC) is a proposed Large Hadron Collider (LHC) accelerator upgrade to increase the machine luminosity by an order of magnitude to $10^{35} \mathrm{~cm}^{-2} \mathrm{~s}^{-1}$. The increased particle fluxes and years of radiation damage means that the Compact Muon Solenoid (CMS) experiment at the LHC will have to replace its entire tracking system in expectation of this. Power consumption is a significant challenge in the design of a future tracker readout system due to requirement for a higher granularity detector. Physics performance must not be compromised so the tracker material contribution should be lowered where possible. It will also be necessary for the tracker to provide some information to the Level 1 system in order to maintain the trigger rate below a maximum of $100 \mathrm{kHz}$. A method of reducing the on-detector data rate for input into a L1 trigger using closely separated ("stacked") pixel layers is presented. Detailed simulations report that a tracking trigger layer would be viable for use at SLHC, reducing the detector data rate by a factor of $\sim 20$ while maintaining a track finding efficiency in excess of $96 \%$ for tracks with $\mathrm{p}_{\mathrm{T}}>2 \mathrm{GeV} / \mathrm{c}$. Two or more stacked layers could be used to reconstruct tracks with $\delta \mathrm{p}_{\mathrm{T}} / \mathrm{p}_{\mathrm{T}}<20 \%$ for $\mathrm{p}_{\mathrm{T}}<20 \mathrm{GeV} / \mathrm{c}$ and with sufficient resolution so as to match tracks with L1 calorimeter objects. Recent progress on stacked module R\&D, trigger simulations and concept designs is presented.

19th International Workshop on Vertex Detectors - VERTEX 2010

June 06 - 11, 2010

Loch Lomond, Scotland, UK

\footnotetext{
* Speaker.

${ }^{\dagger}$ for the CMS collaboration.
} 


\section{The Super-LHC}

The proposed luminosity upgrade[1] for the Large Hadron Collider (LHC) is expected to take place in two phases over a 10 year period after LHC start-up. With an increase of a factor of 10 in luminosity, the LHC experiments will also require various upgrades in order to cope with the increased particle fluxes, data rates and years of radiation damage. The CMS experiment[2] is expected to replace its entire tracking system after an integrated luminosity of $\sim 500 \mathrm{fb}^{-1}$ and its inner pixel detector at least once before this. Aside from the radiation tolerance of sensors and electronics, the most important challenges are the development of low power electronics and power distribution schemes to the front end. To remove increased heat loads within the system, cooling must be improved while tracker material must be reduced in order not to compromise physics performance.

The Level 1 (L1) system[3] is a customised hardware trigger designed to promptly $(<4 \mu \mathrm{s})$ reduce the event rate before event reconstruction and processing on large CPU farms. It has been shown that the L1 trigger suffers from the increased pileup of up to 500 minimum bias interactions per bunch crossing at SLHC[凹]. Raising transverse energy $\left(\mathrm{E}_{\mathrm{T}}\right)$ and momentum thresholds $\left(\mathrm{p}_{\mathrm{T}}\right)$ offer little reduction in rate while adversely affecting sensitivity to low mass discoveries and measurements at the LHC. Since tracking information is not currently used in the L1 trigger decision, it is hoped that its inclusion will stop the trigger exceeding its maximum $100 \mathrm{kHz}$ rate. Providing tracking information to the trigger presents entirely new challenges in the design of an upgraded tracker. Specifically, the on-detector data rate must be reduced significantly for viable readout at the bunch crossing rate of $40 \mathrm{MHz}$. In addition, the tracking trigger must not contribute significantly to the power dissipation and material within the tracker and more importantly must not reduce tracking performance and resolution.

\section{Stacked Tracking Trigger Layers}

Collisions at the LHC are predicted to produce a large number of low momentum particles that make up a significant fraction of hit data generated by the tracker (Figure 1). Charged particles with transverse momentum $\mathrm{p}_{\mathrm{T}}<0.7 \mathrm{GeV} / \mathrm{c}$ are considered uninteresting for the purposes of triggering since they fail to reach the outer sub-detectors due to the bending power of the $4 \mathrm{~T}$ magnetic field.

By correlating hits between closely spaced ("stacked") pixelated sensors, this low $\mathrm{p}_{\mathrm{T}}$ background can be rejected by only selecting hits that lie within a few pixels of each other in the bending plane $(\mathrm{r}-\phi)$. In a $4 \mathrm{~T}$ magnetic field, studies show that for a layer of stacked pixel sensors placed at $25 \mathrm{~cm}$ and a radial separation between sensors of $\sim 1 \mathrm{~mm}$, a pixel pitch of order $100 \mu \mathrm{m}$ in $\mathrm{r}-\phi$ can be used to select tracks with transverse momentum greater than a few $\mathrm{GeV} / \mathrm{c}[5,6$, 6]. In this way, the on-detector data rate can be reduced by at least an order of magnitude before tracking information is forwarded to the L1 trigger for matching to other trigger objects.

Correlation algorithms to match hits between individual sensors and identify high transverse momentum candidates ("stubs") are described in further detail in [6]. Of particular importance is the $r-\phi$ or row correlation window which is the discriminator for measuring the track curvature in the magnetic field. 

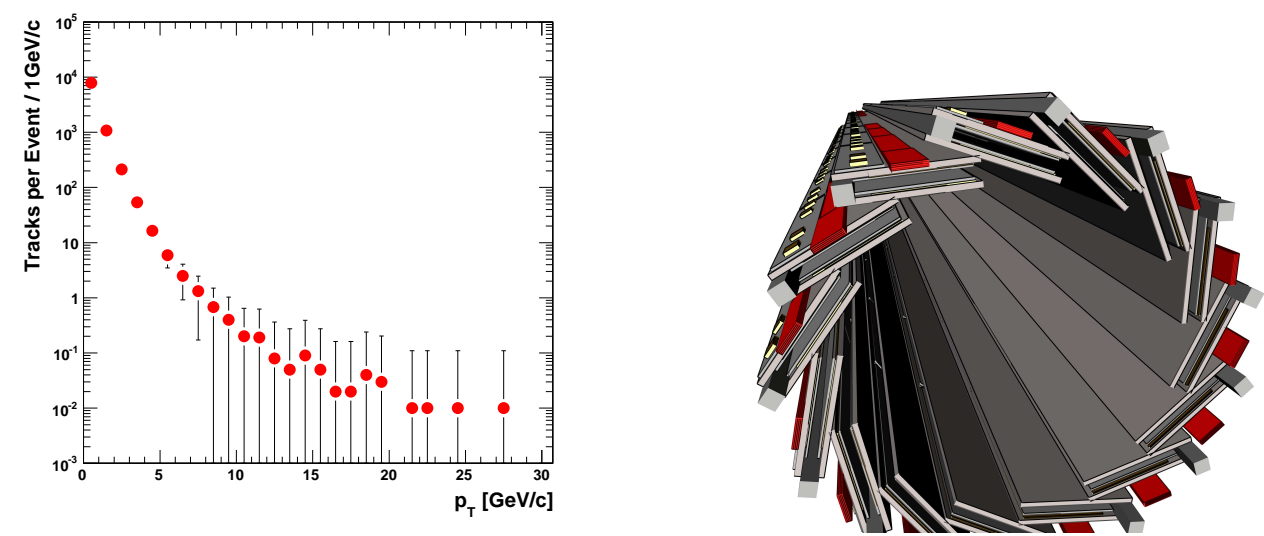

Figure 1: Left: The $\mathrm{p}_{\mathrm{T}}$ spectrum (averaged per event) for all minimum bias particles that leave hits in a sensitive layer placed at a radius of $25 \mathrm{~cm}$ and at an average pileup of $400 \mathrm{p}-\mathrm{p}$ interactions per event. Events were simulated within a magnetic field of $4 \mathrm{~T}$ and a coverage of $|\eta|<2.5$. Right: Visualisation of a GEANT defined stacked pixel layer including cabling, cooling and mechanical support.

\subsection{Simulated Performance of a Single Layer}

Realistic simulations on stacked tracking layers have been performed[6]. The following results were generated with a geometry including two stacked pixel layers at $25 \mathrm{~cm}$ and $35 \mathrm{~cm}$, with full coverage up to $|\eta|<2.5,100 \mu \mathrm{m}$ thick sensors and pixels with $100 \mu \mathrm{m} \times 2.45 \mathrm{~mm}$ pitch in $\mathrm{r} \phi$-z. Long pixels are used in order to minimise power requirements. With this granularity, the occupancy in a typical SLHC event at $25 \mathrm{~cm}$ is expected to be $<1 \%$.
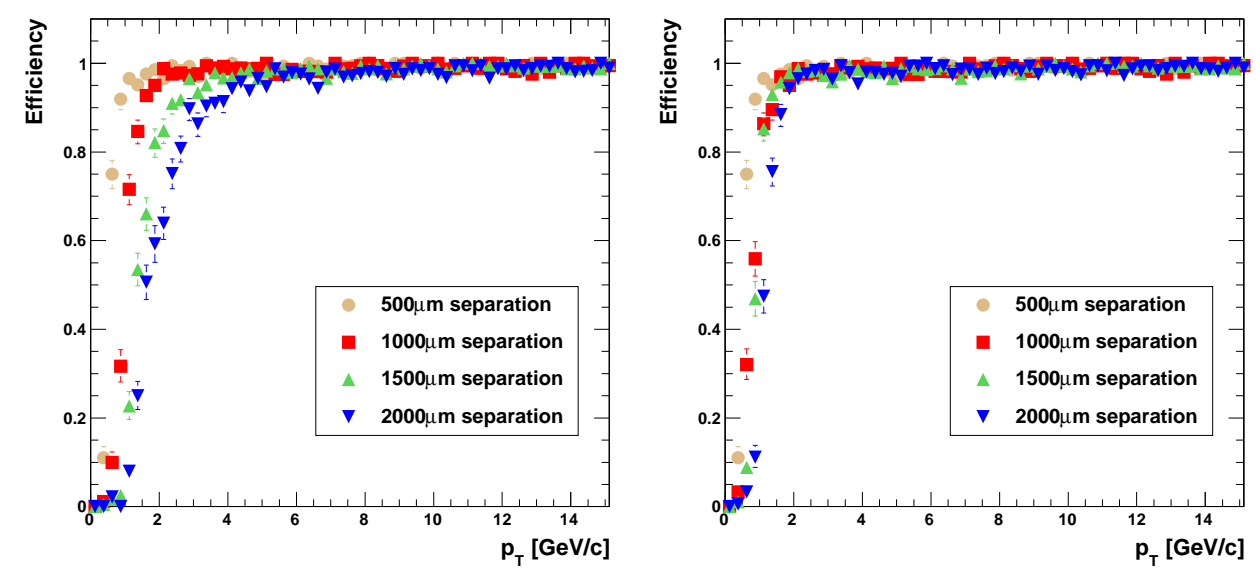

Figure 2: $\mathrm{p}_{\mathrm{T}}$ discrimination performances of a stacked layer for; Left: single $\mu^{ \pm}$tracks at various sensor separations and a fixed 3 pixel row correlation window; Right: single $\mu^{ \pm}$tracks at various sensor separations where the correlation window is widened with sensor separation (see Table 1). All results are for a stacked layer at $25 \mathrm{~cm}$.

For a fixed row correlation window, increasing the sensor separation has the effect of increasing the $\mathrm{p}_{\mathrm{T}}$ cut at which stubs are generated. Figure 目 (left) demonstrates how a stacked layer at 
$25 \mathrm{~cm}$ is expected to perform at discriminating against the transverse momentum of tracks for various sensor separations and a fixed row correlation cut. The result of the simulation using single muons validates those from previous studies[5].

The efficiency $\varepsilon$ described in Figure 2 is defined as the ratio of total number of tracks with Monte Carlo transverse momentum $\left(\mathrm{p}_{\mathrm{T}}\right)$ which generate at least one pixel hit in the stacked pixel layer to the number of tracks with Monte Carlo transverse momentum $\left(\mathrm{p}_{\mathrm{T}}\right)$ which generate at least one stub in the stacked pixel layer.

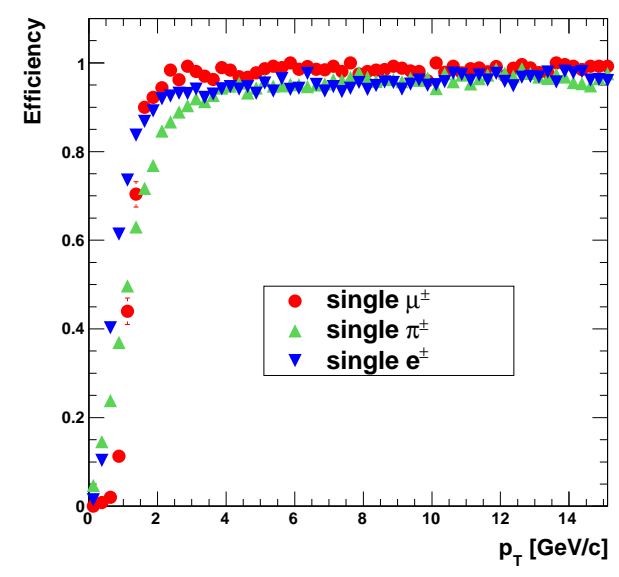

Figure 3: $\mathrm{p}_{\mathrm{T}}$ discrimination performances of a stacked layer for single $\mu^{ \pm}, \pi^{ \pm}$and $\mathrm{e}^{ \pm}$tracks using a layer with a $2 \mathrm{~mm}$ sensor separation and a 5 pixel correlation window. All results are for a stacked layer at $25 \mathrm{~cm}$.

The row correlation window cut is another method of controlling the transverse momentum against which tracks are discriminated. The difference is that while varying the sensor separation modifies the $\mathrm{p}_{\mathrm{T}}$ cut continuously, changing the correlation window will modify the $\mathrm{p}_{\mathrm{T}}$ cut in discrete steps, as defined by the pixel pitch. Figure 2 (right) and Table 2.1 demonstrate that increasing the row window with sensor separation maintains $\mathrm{p}_{\mathrm{T}}$ discrimination performance. Building a layer with a larger sensor separation but with a correlation window that can be varied may be more practical in terms of robustness to triggering demands and the physics at SLHC.

Table 2.1 shows that for a fixed correlation window, a larger separation will increase the effective $\mathrm{p}_{\mathrm{T}}$ cut and therefore reduce the number of generated stubs. However, if tracking isolation is required at $\mathrm{L} 1$, efficient triggering on tracks with transverse momenta of at least $2 \mathrm{GeV} / \mathrm{c}$ [8] will be necessary.The efficiency for triggering of tracks with $\mathrm{p}_{\mathrm{T}}>2 \mathrm{GeV} / \mathrm{c}$ is also provided in Table 2.1 along with the average ratio of duplicate and fake stubs (Figure 4 ) to total stubs. The reduction factor is defined as the ratio of average number of hit pixels to the average number of generated stubs in a layer, per event. It is an indication of the reduction in the number of hits to be read out if correlation was to be performed on detector. An order of magnitude data rate reduction will be required if the readout system is to satisfy existing power and cabling constraints[6].

Figure 3 compares the performance for the stacked layer when selecting muon, pion and electron tracks by $\mathrm{p}_{\mathrm{T}}$. Although the difference appears minimal, the layer is less effective at rejecting low transverse momentum electrons and especially pions, compared to muons. Since the stacked trigger layer assumes an interaction vertex at the beam axis, the $\mathrm{p}_{\mathrm{T}}$ of particles from secondary 


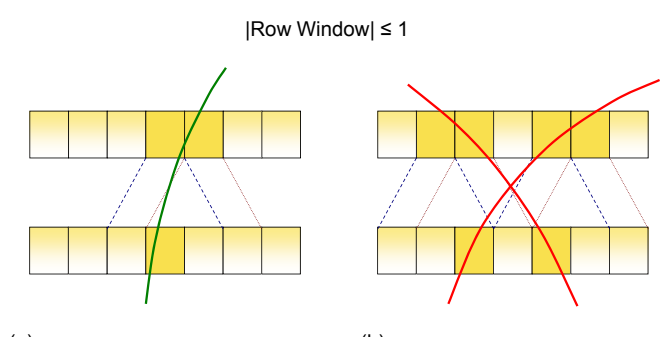

(a)

(b)

Figure 4: Illustration on the origin of duplicate and fake stubs; (a) demonstrates that if the row correlation window is $\geq \pm 1$, clusters of hit pixels can give rise to multiple stubs. The number of duplicates could be reduced to zero using a clustering algorithm either before or after correlation. Tracks which would not normally pass the correlation cut may still produce a stub if hits are incorrectly matched with those from another track (b).

\begin{tabular}{c|c||c|c|c|c|c}
\hline $\begin{array}{c}\text { Sensor } \\
\text { Separation } \\
(\mu \mathrm{m})\end{array}$ & $\begin{array}{c}\text { Row } \\
\text { Window } \\
\text { (pixels) }\end{array}$ & $\begin{array}{c}\varepsilon_{\text {Muon }} \\
\mathrm{p}_{\mathrm{T}}>2 \mathrm{GeV} / \mathrm{c} \\
(\%)\end{array}$ & $\mathrm{N}_{\text {Stubs }}$ & Fake & Duplicate & $\begin{array}{c}\text { Rate } \\
\text { Reduction }\end{array}$ \\
\hline \hline 1000 & 3 & 99.2 & 2670.5 & 6.6 & 30.9 & 22.0 \\
1000 & 4 & 99.2 & 4150.9 & 5.6 & 36.6 & 14.2 \\
2000 & 3 & 97.1 & 1054.1 & 23.3 & 22.4 & 54.4 \\
2000 & 5 & 98.7 & 2248.3 & 18.1 & 28.0 & 25.5 \\
\hline
\end{tabular}

Table 1: Trigger performance of a stacked layer at $25 \mathrm{~cm} . \varepsilon_{\text {Muon }}$ is the efficiency for triggering on $\mu^{ \pm}$tracks with $\mathrm{p}_{\mathrm{T}}>2 \mathrm{GeV} / \mathrm{c}$. The percentage of fake and duplicate stubs and the rate reduction factors are calculated from simulating the stacked layer in minimum bias events under SLHC pileup conditions. A configuration with $2 \mathrm{~mm}$ separation provides adequate performance, while allowing the option of varying the correlation window cut based on operating conditions and physics requirements.

interactions or bremsstrahlung electrons can be reconstructed incorrectly. It is therefore important that the material in the inner detector is minimised.

The trigger algorithm needs to be able to operate efficiently at any luminosity while still offering the same reduction in data output and must also be robust against any local or global fluctuations in occupancy. In the most extreme cases, it may be possible that the stacked layer will be subject to peak hit pixel occupancies of up to $0.63 \% \pm 0.23 \%$ [6]. Figure 5 demonstrate that for occupancies up to $0.6 \%$, the performance of the stacked tracker is robust against pileup.

\subsection{Simulated Performance of a Double Layer}

In a double stack configuration, each layer would be able to provide the necessary data rate reduction required for transmitting tracking information off detector before correlation for track reconstruction. The advantage of this design would be that track $\mathrm{p}_{\mathrm{T}}$ can be measured but no ondetector communication between layers would be needed, removing the need for high bandwidth links and a complex interconnection scheme between modules which greatly increase the power 

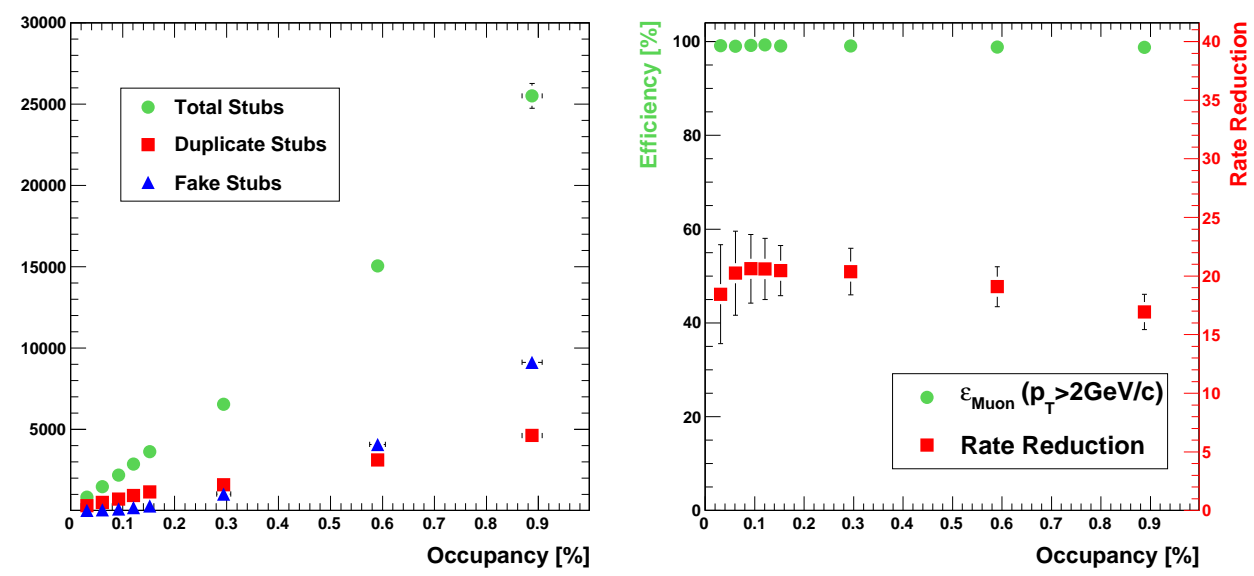

Figure 5: Left: Average number of generated stubs per event and; Right: Trigger efficiency for muons with $\mathrm{p}_{\mathrm{T}}>2 \mathrm{GeV} / \mathrm{c}$ (left scale) and rate reduction factor (right scale) as a function of average layer occupancy. Results are for a stacked layer at $25 \mathrm{~cm}$ with a sensor separation of $1 \mathrm{~mm}$ and a row correlation window of 3 pixels.

consumption and material of the system.

Figure 6 illustrates how tracks are reconstructed. Stubs from the inner stack are successfully correlated if they fall within a $\Delta \phi \Delta \eta=0.02 \times 0.1$ window of the upper seed stub. The window size in $\Delta \phi$ must be large enough to accept low $\mathrm{p}_{\mathrm{T}}$ tracks and to allow for multiple scattering within the inner layers. The $\Delta \eta$ window size is dominated by the size of the interaction region in $\mathrm{z}$. The two stacked layers are placed at radii of $25 \mathrm{~cm}$ and $35 \mathrm{~cm}$ with coverage up to $|\eta|<2.5$. Both layers use $100 \mu \mathrm{m}$ thick sensors with a sensor separation of $2 \mathrm{~mm}$. The transverse momentum is calculated using the two stubs and an assumed vertex at $(0,0)$. This allows a variable cut on $\mathrm{p}_{\mathrm{T}}$ $(\sim 5-50 \mathrm{GeV} / \mathrm{c})$ to be placed on the reconstructed track, as is performed in the current High Level Trigger[3]. Isolation performance for tracks with $2 \mathrm{GeV} / \mathrm{c}$ transverse momenta is not discussed here.

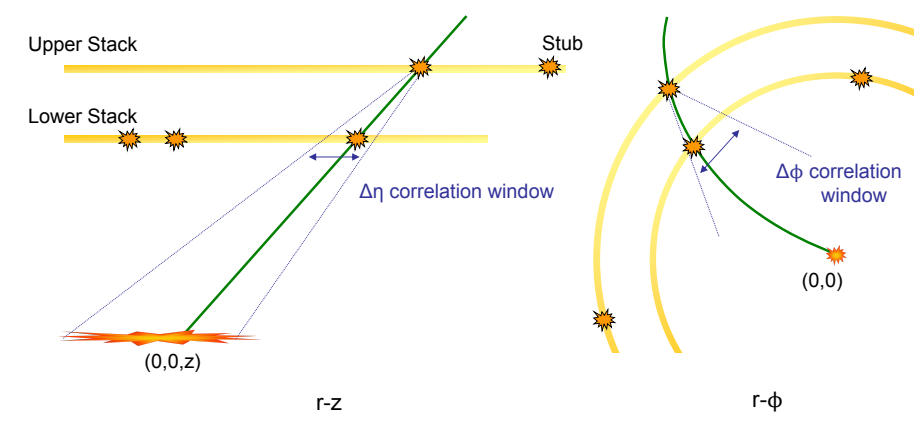

Figure 6: The double stack reconstruction method. Stubs from the inner stacked pixel layer which fall within a $\Delta \eta \Delta \phi$ window of a seed stub in the outer layer are correlated. 


\begin{tabular}{c||c|c|c|c|c}
\hline $\begin{array}{c}\mathrm{p}_{\text {Tcut }} \\
(\mathrm{GeV/c})\end{array}$ & $\begin{array}{c}\varepsilon_{\text {Muon }} \\
(\%)\end{array}$ & $\begin{array}{c}\varepsilon_{\text {Pion }} \\
(\%)\end{array}$ & $\begin{array}{c}\varepsilon_{\text {Electron }} \\
(\%)\end{array}$ & $\mathrm{N}_{\text {Reco }}$ & $\mathrm{N}_{\text {Fake }}$ \\
\hline \hline 4 & 96.9 & 91.8 & 91.5 & 104.6 & 66.6 \\
6 & 97.0 & 91.9 & 89.9 & 55.9 & 43.8 \\
10 & 96.9 & 91.6 & 86.8 & 29.8 & 25.4 \\
\hline
\end{tabular}

Table 2: Trigger performances for single muons, pions and electrons with reconstructed $\mathrm{p}_{\mathrm{T}}>\mathrm{p}_{\mathrm{T} \text { Tut }}$ using the double stack geometry and an individual stack row correlation window cut of 3 pixels. Efficiencies are for reconstructed tracks with Monte Carlo $\mathrm{p}_{\mathrm{T}}$ above $p_{\text {Tcut }}$. Average number of total and fake reconstructed tracks per event obtained under SLHC conditions.

Table 2 shows that the number of reconstructed tracks is much lower than the number of stubs per layer while efficiencies are maintained for muons and pions. Due to electron bremsstrahlung, the electron efficiency falls as the $\mathrm{p}_{\mathrm{T}}$ cut is raised. A large fraction of reconstructed tracks are combinatorial fakes; irreducible without reducing the $\Delta \phi \Delta \eta$ window or supplying additional matching information. However, since the total number of reconstructed tracks is small, this is not expected to be a problem once tracks are matched with calorimeter deposits or muon stubs. Studies measuring the simulated L1 triggering performance for objects such as muons and electrons under SLHC conditions are ongoing.
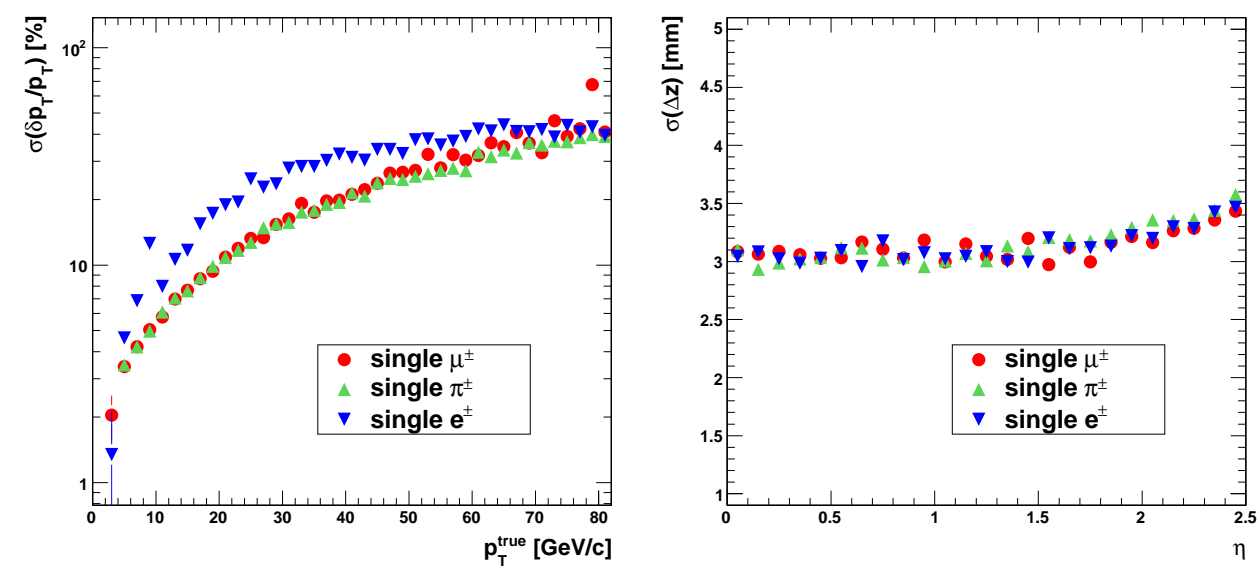

Figure 7: Left: Transverse momentum, and; Right: $\mathrm{z}$ vertex, (rms) resolutions for reconstructed $\mu^{ \pm}, \pi^{ \pm}$ and $\mathrm{e}^{ \pm}$tracks using a double stack layer geometry. Results are for real tracks passing a $4 \mathrm{GeV} / \mathrm{c} \mathrm{p}_{\mathrm{T}}$ cut and a tight correlation window of 3 pixels for the individual stacks.

Figure 7 shows that the transverse momentum resolution is measured to be $<20 \%$ for muons and pions up to $50 \mathrm{GeV} / \mathrm{c}$. Due to bremsstrahlung, the $\mathrm{p}_{\mathrm{T}}$ resolution is slightly worse for electrons. The matching resolution at the calorimeter surface is measured to be $\Delta \phi \Delta \eta<0.02 \times 0.15$. While the $\mathrm{p}_{\mathrm{T}}$ resolution is certainly not acceptable for tracking, it does offer a coarse method for cutting on the transverse momentum so that trigger rates can be reduced if required. It also provides the trigger with an additional cut when matching tracks to calorimeter clusters by calculation of the 
$\mathrm{E}_{\mathrm{T}} / \mathrm{p}_{\mathrm{T}}$ ratio or when matching to muon objects. Increasing the separation between the two stacks would improve the $\mathrm{p}_{\mathrm{T}}$ resolution.

\section{Triggering with Stacked Tracking Layers}

The ultimate objective of the tracking trigger upgrade at CMS is to stop the L1 trigger exceeding its maximum $100 \mathrm{kHz}$ rate. Detailed simulations have begun on identifying the additional triggering performance a system with stacked tracking layers would bring to the current L1 object algorithms. As has been discussed, the triggering efficiency for muons using stacked layers is $\sim 97 \%$ while the $\mathrm{p}_{\mathrm{T}}$ resolution is adequate for matching to the muon system. At high pileup, the muon rate at $\mathrm{L} 1$ is dominated by low $\mathrm{p}_{\mathrm{T}}$ jets which can be easily suppressed if a $\mathrm{p}_{\mathrm{T}}$ matching requirement between the stacked layers and the muon system is imposed.

Of greater importance is the estimated triggering performance of the electron identification algorithms at L1 since the double layer tracking simulations show much poorer efficiencies and momentum resolution. At L1, the electron rate is dominated by high momentum $\pi^{0} \mathrm{~s}$ from jets. It could be expected that matching between stacked layer track candidates and calorimeter deposits might suppress these background rates although without detailed simulations it is impossible to gauge the effect of photon conversions and electron bremsstrahlung on the matching efficiency. Preparatory studies[0] have shown that using two stacked layers to provide a 2-point track plus electromagnetic calorimeter electron candidate, a factor of 20 reduction in rate is possible with respect to the current calorimeter trigger for a given threshold (Figure 8). In this study, the tracks were required to lie within a window $\Delta \phi \Delta \eta<0.25 \times 0.1$ of the calorimeter candidate while both $\mathrm{E}_{\mathrm{T}}$ and $\mathrm{p}_{\mathrm{T}}$ thresholds were placed on the calorimeter candidates and track candidates respectively. With an overall triggering efficiency of $\sim 75 \%$ due to the poor reconstruction performance of the electron track, further studies are under way to determine whether additional tracking layers could provide a better estimate of the electron $\mathrm{p}_{\mathrm{T}}$ despite contributing extra material to the system.

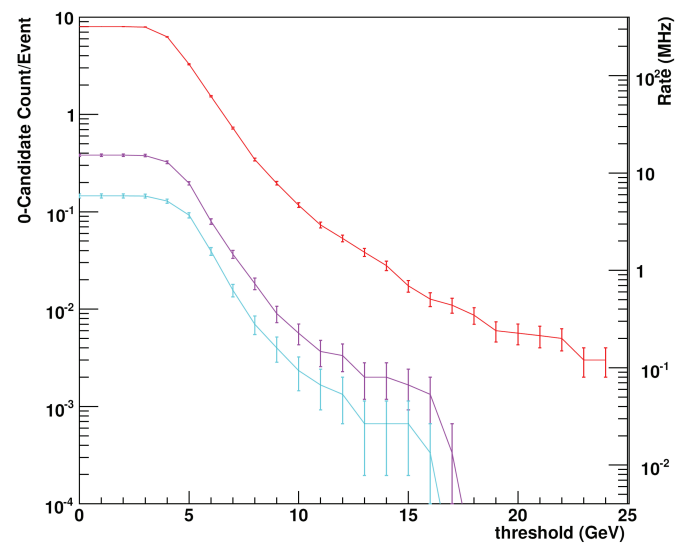

Figure 8: Mean rate of calorimeter candidates (red), 2-point track matched to calorimeter candidates (violet) and 3-point track matched to calorimeter candidates (cyan) as a function of applied $\mathrm{E}_{\mathrm{T}}$ and $\mathrm{p}_{\mathrm{T}}$ thresholds for background events under SLHC conditions ( 200 pileup) [९]. 


\section{Module Concepts for Stacked Tracking Layers}

There are currently a number of stacked layer module concepts under review utilising both existing and pursuing R\&D into novel technologies. The requirement is that such modules must be able to contribute to both triggering at Level 1 while also buffering and transmitting data for readout at $100 \mathrm{kHz}$. This must be achieved with the minimal power penalty while the amount of material in the final system must be reduced with respect to that of the current tracker. Three possible concepts are highlighted here.

The "Edge Readout" design focuses on currently available technologies and the potential for easy prototyping[10]. The module consists of two stacked sensors with a pixel cell size of $\sim 100 \mu \mathrm{m} \times 2 \mathrm{~mm}$ read out by a coarse pitch bump bonded ASIC. Modules of this type would be utilised at intermediate radii $(25-50 \mathrm{~cm})$, making use of the low occupancies so that hits can be transferred to the edge of the chip at low speed and hence low power. At the edge of the module, the readout chips would be interconnected via the PCB. This design allows the possibility to integrate cooling within the inter-sensor gap. The power consumption is estimated to be $<100 \mu \mathrm{W}$ per pixel excluding the link power, which is expected to be of the same order.

The "Vertically Integrated Hybrid Module" is based on 3D-interconnection technology with data transfer between stacked sensors via a $\sim 1 \mathrm{~mm}$ low mass interposer layer[11]. The pixel cell sizes are approximately $100 \mu \mathrm{m} \times 1 \mathrm{~mm}$, with the option of differing pixel lengths between the upper and lower sensors in the module. Analogue data is transferred from the upper sensor through the interposer where a single 3D correlator and readout ASIC resides, on top of the lower sensor. This design allows for the implementation of a flexible local trigger logic with low power consumption per pixel. A 3D demonstrator chip (VICTR) is currently in fabrication.
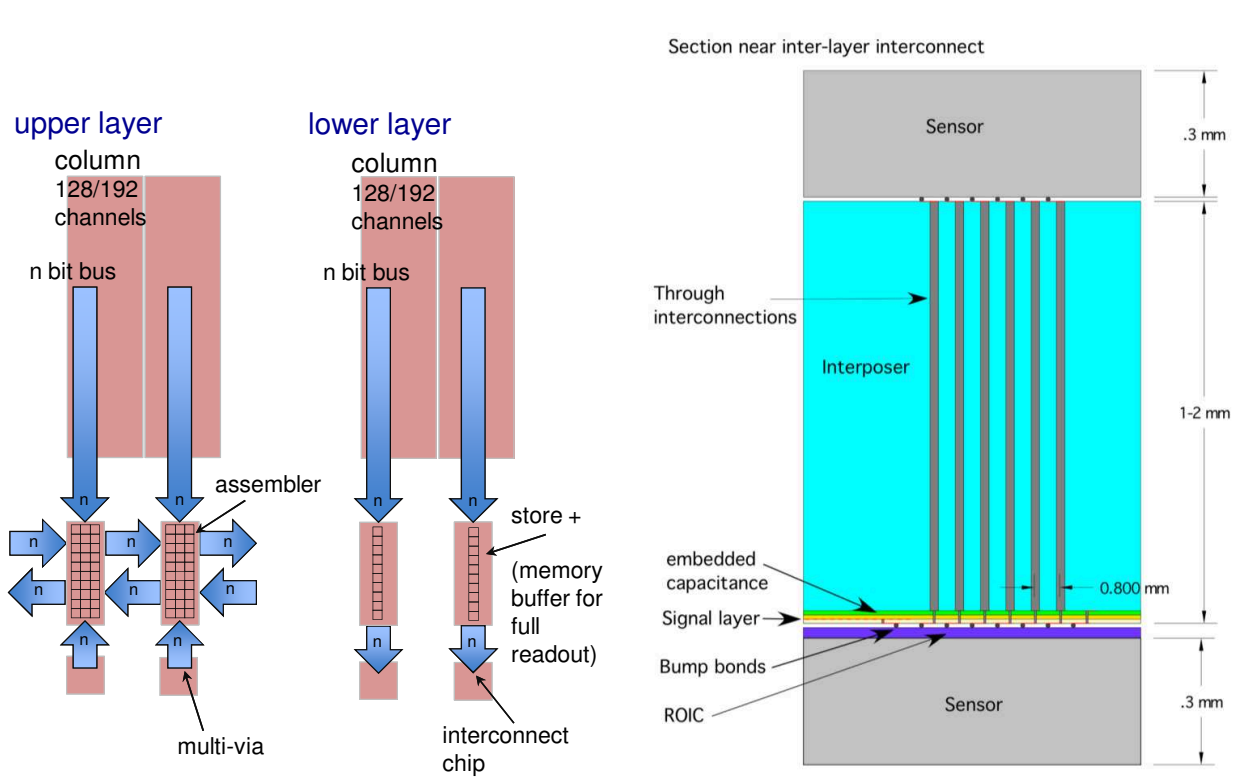

Figure 9: Left: Example illustration of the edge readout module concept, and; Right: a section of the vertically integrated module. 
The "Stacked Strip" module again focusses on currently available technologies but uses layers of closely separated $5 \mathrm{~cm}$ long strip sensors instead of pixels. As such, modules of this type would be used in layers at radii $30-100 \mathrm{~cm}$, and possibly in the endcaps. From both sensors, the strips of pitch 80-100 $\mu \mathrm{m}$ would be wire-bonded at the edges to a binary readout ASIC with a minimum $50 \mu \mathrm{m}$ pitch. A prototype binary chip (CBC) for short strip readout but without triggering capabilities is already in fabrication and future versions could incorporate correlation logic for triggering. This design carries obvious advantages in reducing the complexity of an integrated tracking and triggering system by using the same module and readout ASIC for the entire strip tracker and by using well known and cheap technologies. Further studies are required to determine the viability of such layers for efficient triggering at L1.

\section{Summary}

The CMS experiment plans to upgrade its tracking system in expectation of the LHC luminosity upgrade. The detector design will be driven by the requirements of the unique operating conditions at SLHC, a need to reduce material for improvement in detector and physics performance and the possibilities to provide tracking data to the L1 trigger. The stacked pixel layer concept has demonstrated viability for use at SLHC. Simulations show that a on-detector data reduction of $\sim 20$ with $>96 \%$ efficiency will be possible allowing transfer of data off-detector. Two stacked layers could be used for off-detector reconstruction of tracks with $\delta \mathrm{p}_{\mathrm{T}} / \mathrm{p}_{\mathrm{T}}<20 \%$ for $\mathrm{p}_{\mathrm{T}}<20 \mathrm{GeV} / \mathrm{c}$ and with sufficient resolution so as to match tracks with L1 calorimeter objects. Significant challenges still remain in the realisation of such a system. The power consumption for a single layer is expected to be large and requires careful consideration as do cost and time for prototyping. A variety of module concepts for stacked tracking layers are under consideration and R\&D. The collaboration is working towards defining a common tracker layout in 2011, including layers dedicated to both tracking and providing tracking information to the Level 1 trigger.

\section{References}

[1] F. Zimmermann, CERN Upgrade Plans for the LHC and its Injectors, CERN (2009) CERN-sLHC-PROJECT-Report-0016.

[2] The CMS Collaboration, S. Chatrchyan et al., The CMS experiment at the CERN LHC, JINST 3 (2008) S08004.

[3] The CMS Collaboration, G.L. Bayatian et al., The Trigger and Data Acquisition project, Volume I, The Trigger Systems, CERN CMS TDR 6.1 (2000), CERN/LHCC/2000-038.

[4] The CMS collaboration, CMS Expression of Interest in the SLHC, CERN (2007), CERN/LHCC 2007-014.

[5] J. Jones, G. Hall, C. Foudas, A. Rose, A pixel detector for Level-1 triggering at SLHC, CERN 2005-011 (2005), [hep-ph/0510228].

[6] M. Pesaresi, Development of a new silicon tracker at CMS for Super-LHC, PhD-Thesis, University of London, CERN-THESIS 2010-083 (2010).

[7] A. Bean, The CMS pixel detector and challenges for its upgrade, CERN (2009) CERN-CMS-CR-2009-178. 
[8] The CMS collaboration, M. Pioppi, Tau reconstruction and identification with particle-flow techniques using the CMS detector at LHC, Nuclear Physics B - Proceedings Supplements 189 (2009), p311-316.

[9] A. Rose, The Level-1 Trigger of the CMS experiment at the LHC and the Super-LHC, PhD-Thesis, University of London, CERN-THESIS 2009-141 (2009).

[10] G. Hall, Design of a trigger module for the CMS Tracker at SLHC, in proceedings of Topical Workshop on Electronics for Particle Physics TWEPP2009, (2009).

[11] R. Lipton, 3D Detector and Electronics Integration Technologies: Applications to ILC, SLHC, and beyond, in proceedings of 7th International Hiroshima Symposium on Development and Applications of Semiconductor Tracking Devices, (2009). 